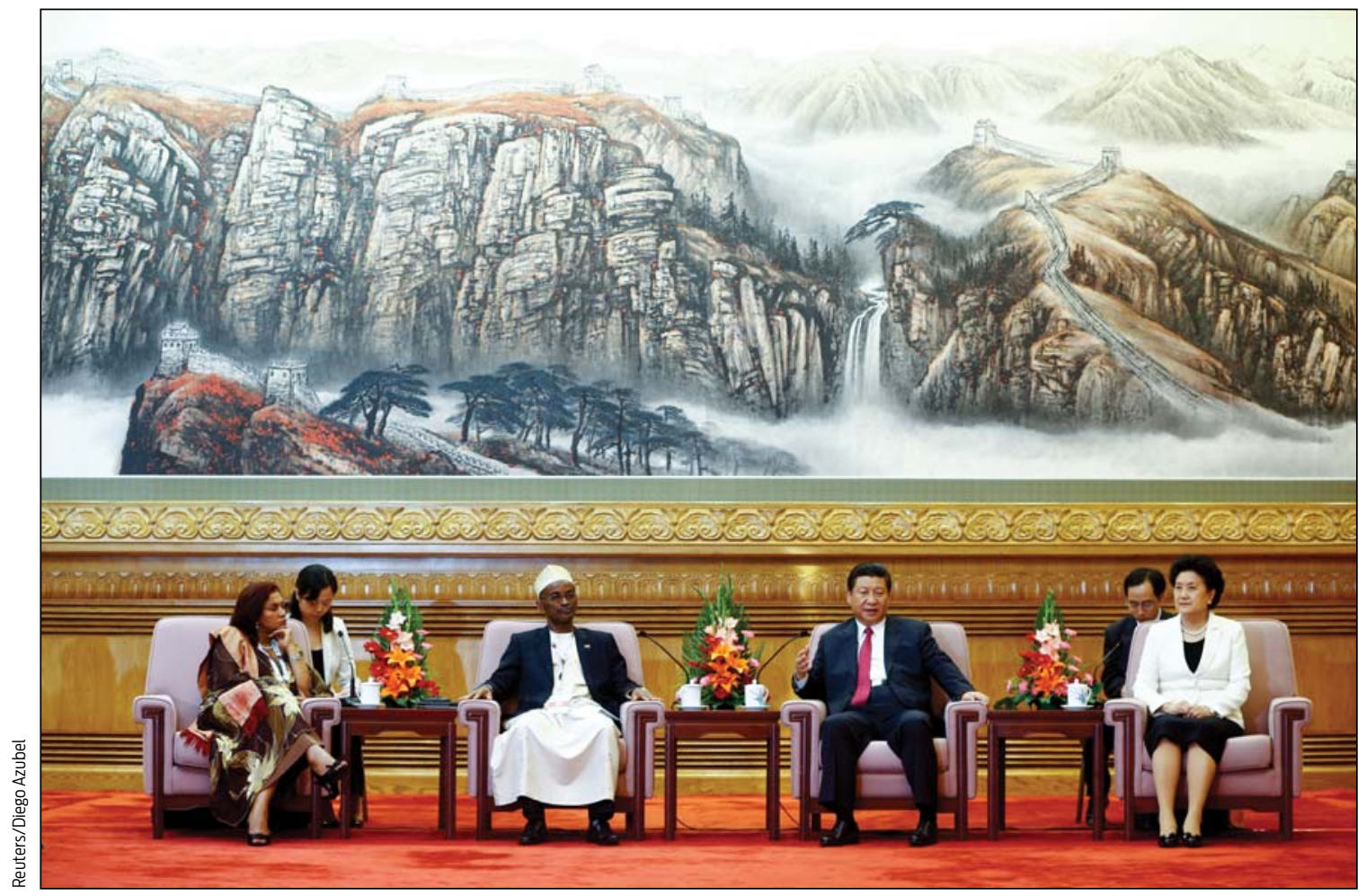

At the opening of the

Ministerial Forum of ChinaAfrica Health Development in the spectacular Great Hall of the People in Beijing are (L-R): Cape Verde Health Minister Dr Maria Cristina Lopez Almeida Fontes Lima, Comoros Vice President Fouad Mohadji, Chinese President Xi Jinping and Chinese Vice Premier Liu Yandong.

\title{
From the CSIRO Digital Productivity and Services Flagship
}

\section{Proactive planning for performance}

As media stories continue about ambulances unable to unload patients because of full hospital beds, overcrowded emergency departments (EDs), and escalating pressures on our health system, frustration is rising for clinicians and ED staff trying to cope with the patient demand coming through the door.

The aim of the recently introduced National Emergency Access Target (NEAT) is that, by $2015,90 \%$ of all patients presenting to an ED will depart from the ED within 4 hours (by being admitted, referred elsewhere or discharged home). The NEAT has been introduced to drive measureable systemic change in removing obstacles to patient flow that contribute to ED overcrowding. It is distinct from the previously used measure of ED flow, access block, which only applied to admitted patients, and it requires a suite of new solutions to improve patient flow. The first report from the National Health Performance Authority on patient time spent in the ED, published in December 2012 (http://www.myhospitals.gov.au/ publications/time-in-emergencydepartment/december-2012/report), shows that most hospitals have a long way to go to achieve the target, with major metropolitan hospitals facing the biggest challenge.

Researchers in health care service delivery from the Commonwealth Scientific and Industrial Research Organisation (CSIRO) are working with hospital clinicians and administrators across Australia to help them meet the requirements of the NEAT. Through analysing hospital datasets, CSIRO researchers can assist in building evidence-driven strategies needed to improve capacity management and identify and ease bottlenecks without compromising

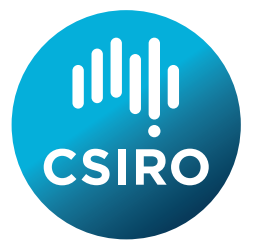

care. An overview of CSIRO's modelling of patient flow, Evidence driven strategies for meeting hospital performance targets (http://www. csiro.au/patientflow), was released in February 2013.

This collaborative research has shown that ED arrivals, admissions and discharges are remarkably predictable. This finding is leading to new solutions that use predicted demand to give increased control of day-to-day operations; for example, proactive bed management and ED planning, and optimisation of inpatient bed configuration.

Patient flow modelling enables a powerful positive culture change, by using site-specific data to drive productivity improvements and giving staff knowledge of likely demand for services. While we cannot control it, we can plan for it - and that has to be a doi: 10.5694/mjal3.10954 


\section{Retinal exam useful for assessing stroke risk}

Patients with hypertensive retinopathy (HR) are at increased risk of stroke, even if their hypertension is well controlled, a study in Hypertension has shown. Data on 2907 patients with hypertension, aged 50 to 73 years at the 1993 to 1995 examination and with no history of diabetes mellitus, stroke or coronary heart disease at baseline, were taken from the Atherosclerosis Risk in Communities Study. A total of 1406 patients (48.4\%) had no signs of HR, 1354 (46.6\%) had mild HR, 146 (5.0\%) had moderate HR and one had severe HR. After a mean follow-up of 13.0 years, the incidence of stroke events for the total population was 0.436 per 100 person-years. In the group with no HR the incidence was 0.322 , for mild HR it was 0.493 , and for moderate HR it was 1.073 per 100 person-years. Additionally, the study found that despite good control of hypertension, those who had mild or moderate HR at baseline were at an increased risk of thromboembolic stroke. "These findings suggest that a retinal examination may be valuable for the assessment of stroke risk in patients with hypertension", the researchers concluded. Hypertension 2013; 12 August (online). doi: 10.1161/hypertensionAHA.113.01414

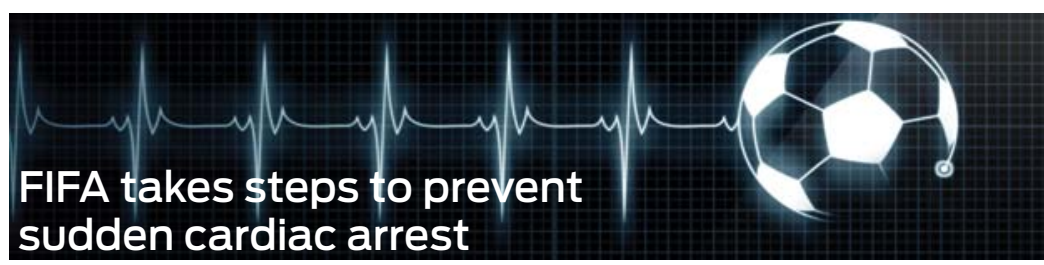

Fédération Internationale de Football Association (FIFA), the international governing body of soccer, has developed an emergency medical bag to deal with on-field emergencies and an 11-step guideline package to help prevent sudden cardiac arrest (SCA), the leading cause of death on the football field. The FIFA Medical Emergency Bag has been distributed to all 209 member associations globally. Its contents, including an automated external defibrillator, are designed to "medically manage a player for approximately $60 \mathrm{~min}$ " with a level of medical care "practically equivalent to an advanced life support level of emergency care", said a consensus statement in the British Journal of Sports Medicine. The FIFA 11 Steps include measures to identify at-risk players through precompetition medical assessments and regular electrocardiograms. Training, equipment, an emergency medical plan, field-of-play medical teams and mandatory ambulances at both training and matches are part of a "comprehensive preventative programme to appropriately manage SCA".

Br J Sports Med 2013; 12 August (online). doi: 10.1136/bjsports-2013-092767

\section{From the MJA archives}

\section{MJA 1951; 7 July (edited extract)}

\section{The mortality in Australia from} cancers peculiar to the female

An attempt has been made to bring out the important features of the mortality of females from cancers of the breast and of the female genital organs, considered at a point of time or over a number of years for evidence of trends. It has been shown that these cancers form an important part of all female mortality and in particular of female cancer mortality. Cancer of the breast and cancer of the ovaries are both increasing in frequency as a cause of death. At some ages there has been, over the last forty years, a decline in the mortality from cancer of the uterus. The effect of conjugal condition on frequency has been noted. It being confirmed that cancer of the ovaries is relatively common among those who have not borne children, cancer of the uterus is more common among the married, and cancer of the breast is more common among the "never married".

H.O. Lancaster School of Public Health and Tropical Medicine Sydney

\section{MDGs should prioritise cervical cancer}

Cervical cancer kills just over 250000 women a year worldwide. Nine out of 10 of those deaths occur in low- and middle-income countries (LMICs), where the mortality rate is $85 \%$. An essay in PLOS Medicine has presented four arguments why the disease should be a priority in the United Nations 2015 Millennium Development Goals (MDGs). First, in many LMICs, women of reproductive age are the main income earners and primary caregivers, meaning their deaths have "profound economic and social consequences". Second, infertility is an outcome of treatment for cervical cancer, and is associated with a higher risk of familial and intimate partner abuse, depression, anxiety and stigmatisation in many LMICs. Third, integration of cervical cancer prevention into HIV, maternal health or reproductive health services using low-cost screening and treatment of preinvasive lesions is feasible in LMICs. Finally, with vaccination now available against human papillomavirus (the cause of almost all cases of cervical cancer), prevention would be relatively simple if vaccination coverage can be scaled up in LMICs. "The post-2015 agenda needs to include a concerted global plan to curb the 'cancer crisis' of developing countries", the authors concluded.

PLOS Med 2013; 13 August (online)

doi: 10.1371/journal.pmed.1001499

\section{Primary care doesn't pay in the US}

In the United States, Medicare reimburses doctors three to five times more for carrying out procedures than for cognitive care services (ie, services fulfilled through the interaction between patient and primary care doctor), even though the latter may conserve costs and promote population health, says a report in JAMA Internal Medicine. The authors conducted an observational study comparing the hourly revenue generated by a physician performing cognitive services with that generated by physicians performing screening colonoscopies and cataract extractions. They found that the revenue from the procedural care generated 368\% (colonoscopies) and 486\% (cataracts) of the revenue from cognitive care. "We demonstrate that 2 common specialty procedures can generate more revenue in 1 to 2 hours of total time than a primary care physician receives for an entire day's work", the authors concluded. "This value discrepancy is a major contributor to the decline in the number of physicians choosing primary care careers."

JAMA Intern Med 2013; 12 August (online)

doi: 10.1001/jamainternmed.2013.9257 